\title{
Asymptotics Beyond All Orders and Analytic Properties of Inverse Laplace Transforms of Solutions
}

\author{
A. Tovbis * \\ Department of Theoretical Mathematics, The Weizmann Institute of Science Rehovot 76100, Israel
}

Received: 1 February 1993/in revised form: 20 December 1993

\begin{abstract}
A number of modern mathematical and physical problems require the study of delicate asymptotic properties lying "beyond" the power series asymptotics. In this paper we suggest a link between these asymptotic problems and some analytic properties of inverse Laplace transforms of the corresponding solutions. The main result claims that these inverse transforms are holomorphic in an appropriately cut complex plane. A direct consequence of this is the nonexistence of solutions to the class of "asymptotics beyond all orders" problems, such as regular shocks of the Kuramoto-Sivashinsky equation ([Gr]), needle crystal solutions of the simple geometrical model of crystal growth ([KS]), solitary wave solutions to a class of the fifth-order Kortveg-de Vries equations ([KO, Sect.8], [GJ]), homoclinic orbits of some singularly perturbed mappings ([Ec, HM]) and others.
\end{abstract}

\section{Introduction}

Let us consider a differential equation of the type

$$
x^{1-r} y^{\prime}(x)=f(x, y), \quad x \in \bar{C}, y \in C^{n}, r \in N,
$$

where the vector-valued function $f(x, y)$ is assumed to be homomorphic at $(\infty, 0) \in$ $\bar{C} \times C^{n}$. The particular equations

$$
\begin{aligned}
& v^{\prime \prime \prime}(x)+v^{\prime}(x)+v^{2}(x)=0, \\
& v^{\prime \prime \prime}(x)+v^{\prime}(x)+\frac{e^{v(x)}}{x}=\frac{1}{x}+\frac{2}{x^{3}},
\end{aligned}
$$

* Supported by a grant of the Ministry of Science and Technology of Israel. Present address: Mathematics Department, West Virginia University, Morgantown, WV 265066310 , USA 
and

$$
v^{\prime \prime \prime \prime}(x)+v^{\prime \prime}(x)+v^{2}(x)=0
$$

that are reducible to (1.1), are considered below.

Everywhere in this paper we will assume that Eq. (1.1) has a formal power series solution

$$
\hat{y}(x)=\sum_{k=1}^{\infty} y_{k} x^{-k / p}, \quad p \in N, n_{0} \in Z .
$$

In what follows without loss of generality we set $p=1$. The word formal means that generally the series (1.5) is divergent, i.e., has zero radius of convergence.

The number $r$ in (1.1) is called the Poincare rank of Eq. (1.1). This equation is said to have the irregular singular point $x=\infty$ if $r>0$. From the general theory of equations with irregular singular points it is known (see [RS]) that for any sector $S$ of opening less than $\pi / r$ the existence of the formal solution (1.5) implies the existence of a proper solution $y(x)$ of (1.1), that is holomorphic in $S$ for sufficiently large $|x|$ and has the asymptotics

$$
y(x) \sim \hat{y}(x), \quad x \rightarrow \infty, \quad x \in S .
$$

Sometimes, if the sector $S$ is sufficiently wide, this asymptotic expansion uniquely determines $y(x)$.

Let some solution $y(x)$ be uniquely determined by the asymptotics (1.6). The considered problem is whether $y(x)$ can possess some symmetry, say, can $y(x)$ be an antisymmetric (odd) function. The answer is definitely negative even if the formal series $\hat{y}(x)$ contains one non-zero even term. However the question becomes "asymptotically beyond all orders" in the case of odd $\hat{y}(x)$. Then a natural approach (see [KS, Gr, HM]) is to find exponentially small terms of $y(x)$, lying beyond the power series asymptotics while $x \in S$ (hidden exponents), and to examine whether they are odd or not.

The essence of the problem here is to evaluate the prefactors of the hidden exponents which are known as the Stokes constants. In [T2] we show that in fact the Stokes constants are "generalized" residues of the corresponding inverse Laplace transforms $Y(p)$ of $y(x)$. Thus we get explicit formulas for the Stokes constants in terms of $Y(p)$. Subsequently we show that in a certain particular case, one can use formal Borel summation to evaluate the Stokes constants.

It turns out, however, that the evaluation of the Stokes constants is not a necessary stage in the search for symmetries of $y(x)$. Instead, in this paper we show that if $y(x)$ is indeed a symmetric or, respectively, an antisymmetric function then the asymptotics (1.6) is valid in so wide a sector such that the formal series $\hat{y}(x)$ must be convergent. Therefore, in the class of problems under consideration, the existence of a solution with some symmetry is equivalent to the convergence of the formal power series solution.

Thus the existence of an antisymmetric solution of Eq. (1.2) and symmetric solutions of Eq. (1.3), (1.4), having the formal power series asymptotics

$$
\begin{aligned}
& \hat{v}(x) \sim \frac{1}{x}-\frac{6}{x^{3}}+\frac{132}{x^{5}}+\cdots, \\
& \hat{v}(x) \sim-\frac{2}{x^{2}}+\frac{50}{3} x^{-4}-\frac{6104}{15} x^{-6}+\cdots,
\end{aligned}
$$


and

$$
\hat{v}(x) \sim-\frac{6}{x^{2}}+\frac{90}{x^{4}}-\frac{2790}{x^{6}}+\cdots
$$

respectively, implies the convergence of (1.7), (1.8) and (1.9). However these series are divergent (see asymptotic formulas for the coefficients of $\hat{v}(x)$ in [GJ, Gr]; also see below). The obtained contradictions serve to prove the nonexistence of solutions of Eq. (1.2)-(1.4) with the required symmetries. This is the essential part of the demonstration that for a sufficiently small positive $\varepsilon$ the reduced KuramotoSivashinsky equation

$$
\varepsilon^{2} v^{\prime \prime \prime}+\left(1-4 \varepsilon^{2}\right) v^{\prime}=1-v^{2}
$$

has no regular shock solutions $([\mathrm{Gr}])$, the simple geometric model of crystal growth

$$
\varepsilon^{2} v^{\prime \prime \prime}+v^{\prime}=\cos v
$$

has no needle crystal solutions ([KS]) and the reduced fifth-order Kortveg-de Vries equation

$$
\varepsilon^{2} v^{\prime \prime \prime \prime}+v^{\prime \prime}+v^{2}-c v=0, c \in R^{+}
$$

has no travelling wave solutions ([GJ]) and no homoclinic orbits. (The latter problems were studied in [KO, Ec] by different methods.) The proposed approach is also applicable to singularly perturbed difference equations, for example to the problem of exponentially small splitting of separatrices of a standard mapping (see [HM]).

The divergence of formal series (1.7)-(1.9) can be rigorously shown using the sign alternation of these series. Consider, for instance, Eq. (1.4). The convergence of its formal solution (1.9) implies the existence of an entire solution $V(p)$ to the convolution equation

$$
\left(p^{4}+p^{2}\right) V(p)+V(p) * V(p)=0,
$$

which is the inverse Laplace transform of (1.4). Suppose $V(p)$ is an entire function. Then $V(i) * V(i)=0$. At the same time the sign alternation of (1.9) ([GJ]) implies that $i V(p)$ is real valued and positive along the positive imaginary semi-axis, so $V(i) * V(i) \neq 0$. The obtained contradiction proves the divergence of (1.9).

Let us provide some comments concerning the mentioned singularly perturbed equations on the example of (1.11). A needle crystal solution of (1.11) is a solution that satisfies the boundary conditions $v(s, \varepsilon) \rightarrow \pm \frac{\pi}{2}$ as $s \rightarrow \infty$. The unperturbed equation $v^{\prime}=\cos v$ has a needle crystal solution $v(s, 0)=-\frac{\pi}{2}+2 \tan ^{-1} e^{s}$. The problem is whether this needle crystal solution survives the singular perturbation.

In fact, this problem has been reduced to the existence of the symmetric solution to (1.3), which possesses the asymptotics (1.8) in the left half-plane. (By the same reason one could consider the right half-plane instead of the left.) The reduction requires the technique of matching of properly defined outer and inner asymptotics and is one of the principal points of the pioneering work [KS]. Unfortunately the framework of this paper does not allow us to present this reduction. Let us only mention here that the outer $\sum_{k=0}^{\infty} \varepsilon^{2 k} v_{k}(s)$ and the inner $-\frac{\pi}{2}+i \ln \left(\frac{2}{\varepsilon}\right)+i \sum_{k=0}^{\infty} \varepsilon^{2 k} \tilde{v}_{k}(x)$ asymptotics of $v(s, \varepsilon)$ are matching near the pole $s=\frac{\pi}{2} i$ of the unperturbed solution 
$v(s, 0)=v_{0}(s)$, where $s=\frac{\pi}{2} i+\varepsilon x$. Then $\tilde{v}_{0}(x)+\ln (-x)$ satisfies (1.3) and possesses the asymptotics (1.8) in the left half-plane.

The following section contains a number of the necessary assertions from the theory of ordinary differential equations with irregular singularities. In Sect. III we formulate the Main Theorem (Theorem 3) on Laplace originals and show the nonexistence of solutions to (1.2)-(1.4) with the required properties. Sect. IV contains the proof of Theorem 3 .

The author is very grateful to Roger Grimshaw for attracting his attention to the subject.

\section{Nonlinear ODE's with Irregular Singularities}

The existence of the formal solution (1.5) of Eq. (1.1) implies that $f(\infty, 0)=0$. Let us represent (1.1) as

$$
y^{\prime}(x)=x^{r-1}(\Lambda y(x)+\tilde{f}(x, y(x)))
$$

where $\Lambda=\frac{\partial f}{\partial y}(\infty, 0)$ and $\tilde{f}(t, y)=f(t, y)-\Lambda y$. Then

$$
\tilde{f}(\infty, 0)=0, \quad \frac{\partial \tilde{f}}{\partial y}(\infty, 0)=0 .
$$

Equation (1.1) is called nondegenerate if $\Lambda$ is a nondegenerate matrix. In the opposite case it is called degenerate. The nondegenerate equations were considered in [Wa]. Our study of a degenerate equation in [T1] is based on the reduction to an equation of the "type" (2.1) with an invertible matrix $\Lambda$. Speaking more rigorously, we showed there that any equation (1.1) which possesses the formal solution (1.5) is reducible to

$$
y^{\prime}(x)=x^{R-I}(\Lambda y(x)+\tilde{f}(x, y(x)))
$$

by means of the transformations

$$
y(x) \mapsto y(x)-\sum_{k=1}^{n_{1}} y_{k} x^{-k}, \quad y(x) \mapsto x^{-n_{2}} T(x) y(x),
$$

where $n_{1}, n_{2}$ are appropriate natural numbers and $T(x)$ is an appropriate matrix polynomial, such that $\operatorname{det} T(x) \not \equiv 0$. In Eq. (2.3) $\mathrm{I}$ is the identity matrix, $R$ and $\Lambda$ are diagonal matrices with the entries

$$
r_{j} \in Q \cap[0, r], \quad \lambda_{j} \in C \backslash\{0\}, \quad j=1, \ldots, n,
$$

respectively, where $\operatorname{Re} \lambda_{j}>0$ if $r_{j}=0$. The vector-valued function $\tilde{f}(x, y)$ is holomorphic at $(\infty, 0) \in \bar{C} \times C^{n}$ and satisfies (2.2).

Let $J$ denote the set of indicies $\left\{j: r_{j} \neq 0\right\}$.

Definition 1. The exponentials $e^{\frac{\lambda_{j}}{r_{j}} x^{r_{j}}}, j \in J$, are called characteristic exponentials of (1.1). 
The characteristic exponentials are uniquely determined by Eq. (1.1) and its formal solution (1.5). For example, $e^{ \pm i x}$ are the characteristic exponentials of both (1.2) and (1.3).

Theorem 1 ([RS]). If (1.1) has a formal power series solution (1.5) then for any sector $S$ of opening less than $\pi / r$ there exists a number $x_{s}>0$ and a proper solution $y(x)$ of (1.1) so that $y(x)$ is holomorphic in $S \cap\left\{x:|x| \geqq x_{s}\right\}$ and $y(x)$ has the asymptotic expansion (1.6) in $S$.

Let $\Psi_{S}$ denote the set of all solutions of (1.1) described in Theorem 1 .

Theorem 2 ([T1]). The dimension $d_{S}$ of the manifold of solutions $\Psi_{S}$ is equal to the number of the characteristic exponentials decreasing in the whole $S$ as $x \rightarrow \infty$. If $d_{S}=0$ then $\Psi_{S}$ contains no more than one solution.

Definition 2. $A$ ray $\tau$ on the Riemann surface of $\ln x$ is called a Stokes ray of (1.1) if at least one of the characteristic exponentials is oscillating along $\tau$.

Remark 1. Let $\tilde{S}$ be an open sector and $S$ be the extension of $\tilde{S}$ in both directions up to the nearest Stokes rays. Then it is well known that $\Psi_{\tilde{S}}=\Psi_{S}$, i.e., only a Stokes ray is the lateral bound of validity of the asymptotics (1.6).

Definition 3. Equation (1.1) is called a one-level equation of order $v$ if $R \neq 0$ and if all the characteristic exponentials of (1.1) are of exponential order $v$.

Equations (1.2)-(1.4), for instance, are one-level equations of order 1. The following well known assertion is a direct consequence of Theorem 2 .

Corollary 1. If (1.1) is a one-level equation of order $v$ and if for some sector $S$ of opening greater than $\frac{3 \pi}{v}$ the set $\Psi_{S} \neq \emptyset$, then the formal power series (1.5) is convergent.

Indeed, let for the sake of simplicity $v=1$. If in the sector $S y(x)=y\left(x e^{2 \pi i}\right)$, then $y(x)$ is holomorphic at infinity (see, for instance, [Wa], Th. 8.6). Otherwise, let the $\tilde{S}$ on the complex $x$-plane be the intersection of the projections of $S$ from different leaves. Then the opening of $\tilde{S}$ is greater than $\pi$ and $y(x), y\left(x e^{2 \pi i}\right) \in \Psi_{\tilde{S}}$. This contradicts Theorem 2 .

\section{Nonexistence of Solutions of (1.2)-(1.4) with the Required Symmetries}

Let $\tau$ be a Stokes ray of the one-level equation (1.1) of order $v \in N$. Let $\varepsilon>0$ be so small that no other Stokes rays lie between $\tau$ and $\tau e^{-i \varepsilon}$. By $\tilde{S}$ we denote the open sector bounded by the rays $\tau e^{-i \frac{\varepsilon}{2}}$ and $\tau e^{i\left(\frac{\pi}{v}-\varepsilon\right)}$. Then $\Psi_{\tilde{S}} \neq \emptyset$ according to Theorem 1. Moreover, $\Psi_{\tilde{S}}=\Psi_{S}$, where the sector $S \supset \tilde{S}$ is bounded by the rays $\tau e^{-i \varepsilon}, \tau e^{i \frac{\pi}{v}}$. Then $\Psi_{s} \neq \emptyset$ and $\Psi_{S}$, according to Theorem 2, contains a unique solution $y(x)$. In what follows without loss of generality we assume that $S$ is bisected by the positive real semiaxis. This implies that $\arg \lambda_{j} \neq 0$ for any $j \in J$. 
Let $\sqrt[v]{-\frac{\lambda_{j}}{v}}=\left|\frac{\lambda_{j}}{v}\right|^{\frac{1}{v}} e^{\frac{l}{v}\left(\arg \frac{\lambda_{j}}{v}-\pi\right)}$. By $U$ we denote the complex $p$-plane with the cuts from points $\sqrt[v]{-\frac{\lambda_{j}}{v}} e^{2 i k \frac{\pi}{v}}$, where $j \in J, k=0, \ldots, v-1$, in the directions $\arg p=$ $\frac{1}{v}\left(\arg \frac{\lambda_{j}}{v}+(2 k-1) \pi\right)$ respectively.

In what follows we use the standard direct and inverse $v$-Laplace transforms defined by the formulas

$$
\left(\mathscr{L}_{v} V(p)\right)(x)=v \int_{0}^{\infty} e^{-x^{v} p^{v}} p^{\nu-1} V(p) d p
$$

and

$$
\left(\mathscr{L}_{v}^{-1} v(x)\right)(p)=\frac{v}{2 \pi i} \int_{\gamma} e^{p^{v} x^{v}} x^{\nu-1} v(x) d x
$$

respectively. Here the contour $\gamma$ is obtained from the vertical line, which is situated in the right half-plane, by means of the transformation $x^{1 / v}$. Note that $\mathscr{L}^{ \pm 1}=\mathscr{L}_{1}^{ \pm 1}$ are the usual direct and inverse Laplace transforms.

Theorem 3 (Main Theorem). Let (1.1) be a one-level equation of order $v$ and let $y \in \Psi_{s}$. Then $\left(\mathscr{L}_{v}^{-1} y\right)(p)$ is holomorphic in $U$.

Let us now utilize Theorems $1-3$ to show that the solution $v(x)$ of (1.2) defined by its asymptotics (1.7) in the right half-plane cannot be purely imaginary-valued on any segment of the imaginary axis (and therefore cannot be antisymmetric).

1. The characteristic exponentials of (1.2) are $e^{ \pm i x}$. Then according to Theorems 1,2 the solution $v(x)$ exists and is uniquely determined. Moreover, it possesses asymptotics (1.7) in the sector $|\arg x|<\pi$ according to Remark 1 .

2. Suppose $v(x)$ is purely imaginary-valued on some segment, say, of negative imaginary semiaxis. Let us consider the complex $x$-plane with the cut along the positive imaginary semiaxis. Then according to the reflection principle $v(x)$ possesses the asymptotics (1.7) in the sector $-\frac{3 \pi}{2}<\arg x<\frac{\pi}{2}$. This sector can be enlarged to $-2 \pi<\arg x<\pi$ by means of Remark 1 .

3. According to Theorem $3\left(\mathscr{L}_{v}^{-1} v\right)(p)$ is holomorphic at the origin of the $p$ plane. Then, one can use the known relation between the power series asymptotics of $v(x)$ and the Taylor expansion of $\left(\mathscr{L}_{v}^{-1} v\right)(p)$ at the origin to demonstrate that all the mentioned Taylor coefficients are real. So $\left(\mathscr{L}_{v}^{-1} v\right)(p)$ and thus $v(x)$ are realvalued on the corresponding positive real semiaxes.

4. The reflection principle can be utilized now once more to show that $v(x)$ possesses the asymptotics (1.7) in the sector $|\arg x|<2 \pi$. Then according to Corollary 1 the series (1.7) is convergent. The obtained contradiction shows the nonexistence of the antisymmetric solution to (1.2), which possesses the asymptotics (1.7) in the right half-plane.

The arguments mentioned above could be as well applied to Eq. (1.3), (1.4). One can get a number of generalizations of this scheme to different problems, including differential, difference and possibly some other classes of equations. 


\section{Proof of Theorem 3}

1. The General Approach. We first present the proof under the assumption that $v=1$ and Eq. (1.1) has $n$ characteristic exponentials $e^{\lambda_{j} x}, j=1, \ldots, n$. The case $v>1$ and the degenerate case (when the number of characteristic exponentials is less than $n$ ) are considered later.

The holomorphic vector-valued function $\tilde{f}(x, y(x))$ can be expanded in the uniformly and absolutely convergent series

$$
\tilde{f}(x, y)=\sum_{|\alpha| \geqq 0} \tilde{f}_{\alpha}(x) y^{\alpha}(x),
$$

in some neighborhood of $x=\infty$. Here $\alpha$ is a multi-index. Then the series

$$
\tilde{F}(p, Y(p))=\sum_{|\alpha| \geqq 0} \tilde{F}_{\alpha}(p) * Y^{* \alpha}(p)
$$

converges absolutely and uniformly on any compact subset of $R^{+}$in the complex $p$-plane. Here the convolution is defined as $V_{1} * V_{2}(p)=\int_{0}^{p} V_{1}(p-\tau) V_{2}(\tau) d \tau, Y^{* \alpha}$ denotes the $\alpha^{\text {th }}$ convolution power and

$$
Y(p)=\left(\mathscr{L}^{-1} y\right)(p), \quad \tilde{F}_{\alpha}(p)=\left(\mathscr{L}^{-1} \tilde{f}_{\alpha}\right)(p) .
$$

Therefore we can define $\mathscr{L}^{-1}[\tilde{f}(x, y(x))]$ as $\tilde{F}(p, Y(p))$.

Here is the idea of the following proof: we represent

$$
Y(p)=\sum_{k=1}^{\infty} \Delta Y_{k}(p)
$$

and construct a majorizing series

$$
Z(p)=\sum_{k=1}^{\infty} \Delta Z_{k}(p)
$$

which converges uniformly on any compact subset of $U$.

2. Properties of $Z(p)$. The coefficients $\tilde{f}_{\alpha}(x)$ in (4.1) are holomorphic at infinity. Therefore they can be expanded in the Taylor series

$$
\tilde{f}_{\alpha}(x)=\sum_{k=0}^{\infty} \tilde{f}_{\alpha, k} x^{-k}, \quad|\alpha| \geqq 0 .
$$

Let us define

$$
\hat{f}_{\alpha}(x)=\sum_{k=0}^{\infty}\left|\tilde{f}_{\alpha, k}\right| x^{-k}, \quad|\alpha| \geqq 0,
$$

and

$$
\hat{f}(x, z)=\sum_{|\alpha| \geqq 0} \hat{f}_{\alpha}(x) z^{\alpha}, \quad z \in C^{n}
$$


Here the entries of the vector $|C|$ are the absolute values of the corresponding entries of the vector $C$. Then the vector-valued function $\hat{f}(x, z)$ is holomorphic at $(\infty, 0) \in \bar{C} \times \bar{C}^{n}$. According to (2.2) we get

$$
\hat{f}(\infty, 0)=0, \quad \frac{\partial \hat{f}}{\partial z}(\infty, 0)=0 .
$$

Let us represent $U=\bigcup_{\delta>0} U_{\delta}$, where $U_{\delta}$ consists of all points $p$ such that the segment $[0, p]$ does not intersect any disk $\left|\lambda_{k}+\tau\right| \leqq \delta, k=1, \ldots, n$. Then if $Y(p)$ is holomorphic in any $U_{\delta}$ then it is holomorphic in $U$.

Let us define $Z(p)$ by

$$
Z(p)=\frac{1}{\delta} \hat{F}(p, Z(p)),
$$

where $\hat{F}(p, Z(p))=\sum \hat{F}_{\alpha}(p) * Z_{(p)}^{* \alpha}, \hat{F}_{\alpha}=\mathscr{L}^{-1} \hat{f}_{\alpha}$. Let us also assume $Z_{0}(p) \equiv 0$, $Z_{n+1}(p)=\frac{1}{\delta} \hat{F}\left(p, Z_{n}(p)\right), n \in N$.

Lemma 1. Equation (4.7) has a unique entire solution given by (4.4), where $\Delta Z_{k+1}(p)=Z_{k+1}(p)-Z_{k}(p)$. The series (4.4) converges uniformly on any compact subset of $C$. All $\Delta Z_{k}(p)$ are entire vector-valued functions and their vector Taylor coefficients at the origin consists of nonnegative real numbers.

Proof. The direct Laplace transform transfer (4.7) into

$$
z(x)=\frac{1}{\delta} \hat{f}(x, z(x))
$$

where $z(x)=(\mathscr{L} Z)(x)$. Then according to the implicit function theorem and to (4.6), Eq. (4.8) has a unique solution $z(x)$. This solution is holomorphic at infinity and $z(\infty)=0$. So, according to the standard fixed point method $z(x)$ can be represented

$$
z(x)=\sum_{k=1}^{\infty} \Delta z_{k}(x),
$$

where $\Delta z_{k+1}=z_{k+1}-z_{k}, z_{k+1}=\frac{1}{\delta} \hat{f}\left(x, z_{k}\right), k \in N$, and $z_{0} \equiv 0$. The series converges uniformly in some neighborhood at infinity. Now all the required properties of the series (4.4) can be immediately derived from (4.9).

3. The Majorization of $Y(p)$. The inverse Laplace transform transfers Eq. (2.1) into

$$
Y(p)=-(I p+\Lambda)^{-1} \tilde{F}(p, Y(p)),
$$

where $I$ is the identity matrix. Let us denote $\Delta Y_{k+1}=Y_{k+1}-Y_{k}$, where $Y_{0}(p) \equiv 0$ and

$$
Y_{k+1}(p)=-(I p+\Lambda)^{-1} \tilde{F}\left(p, Y_{k}(p)\right), \quad k \in N .
$$

Lemma 2. For any $k \in N$ the vector-valued functions $\Delta Y_{k+1}(p)$ are holomorphic in $U_{\delta}$ and

$$
\left|\Delta Y_{k+1}(p)\right|<\Delta Z_{k+1}(|p|)
$$

Proof. For $k=0$ the statements follow from the definitions of $\Delta Y, \Delta Z$. Let us prove these statements for some $k+1$ providing their validity for $1, \ldots, k$. To this end we need the two following lemmas. 
Lemma 3. Let $n \in N, \sigma \in R^{+}$and functions $V_{i} \in C[0, \sigma], W_{i} \in C[0, \sigma], i=1, \ldots, n$. Then if for all $i$ and for all $\tau \in[0, \sigma]$ we have $\left|V_{i}(\tau)\right| \leqq W_{i}(\tau)$, then

$$
\left|V_{1} * \cdots * V_{n}(\sigma)\right| \leqq W_{1} * \cdots * W_{n}(\sigma)
$$

The proof can be given by induction by $n$.

Lemma 4. Let the entries of two pairs of n-dimensional vector-valued functions $V_{1}(\tau), W_{1}(\tau)$ and $V_{2}(\tau), W_{2}(\tau)$ satisfy the conditions of Lemma 3. Moreover, let $|\Delta V(\tau)| \leqq \Delta W(\tau)$ for all $\tau \in[0, \sigma]$, where $\Delta V=V_{2}-V_{1}, \Delta W=W_{2}-W_{1}$, and where the vector inequality holds elementwise.

Then

$$
|\Delta \tilde{F}(\sigma, V)| \leqq \Delta \hat{F}(\sigma, W)
$$

where

$$
\Delta \tilde{F}(\sigma, V)=\tilde{F}\left(\sigma, V_{2}\right)-\tilde{F}\left(\sigma, V_{1}\right), \quad \Delta \hat{F}(\sigma, W)=\hat{F}\left(\sigma, W_{2}\right)-\hat{F}\left(\sigma, W_{1}\right) .
$$

Proof. We can make a $C$-continuation of $W_{2}(\sigma)$ on the whole $R^{+}$so that $w_{2}(x)=$ $\left(\mathscr{L} W_{2}\right)(x)$ is defined and is of the order $O\left(x^{-1}\right)$ as $x \rightarrow \infty$ in the right half-plane. Since $\hat{f}(x, z)$ is holomorphic at $(\infty, 0) \in \bar{C} \times \bar{C}^{n}$, hence we can represent

$$
\left(\mathscr{L}^{-1} \hat{f}\left(x, w_{2}(x)\right)\right)(\sigma)=\hat{F}\left(\sigma, W_{2}(\sigma)\right)=\sum_{|\alpha| \geqq 0} \hat{F}_{\alpha}(\sigma) * W_{2}^{* \alpha}(\sigma) .
$$

The series (4.13) is convergent for any $\sigma \geqq 0$. Therefore it is enough to prove (4.12) for each term of (4.13) independently.

Let us define $w_{1}(x)=\left(\mathscr{L} W_{1}\right)(x)$ in the same way as $w_{2}(x)$. Let $\alpha$ be a multiindex $\alpha=\left(\alpha_{1}, \ldots, \alpha_{m}\right)$. Then

$$
\begin{aligned}
\Delta w^{\alpha}= & w_{2}^{\alpha}-w_{1}^{\alpha}=\sum_{j=1}^{m} \prod_{k=0}^{j-1}\left(w_{2}^{(k)}\right)^{\alpha_{k}} \cdot \prod_{k=j+1}^{m}\left(w_{1}^{(k)}\right)^{\alpha_{k}} \\
& \times \sum_{\ell=0}^{\alpha_{j}-1}\left(w_{2}^{(j)}\right)^{\alpha_{j}-\ell-1}\left(w_{1}^{(j)}\right)^{\ell}\left(w_{2}^{(j)}-w_{1}^{(j)}\right)=g_{\alpha}\left(w_{1}, w_{2}\right) \Delta w,
\end{aligned}
$$

where $w^{(j)}$ means the $j^{\text {th }}$ entry of the column vector $w$ and $g_{\alpha}$ is the corresponding row vector.

That means that we can represent

$$
\begin{gathered}
\Delta W^{\alpha}=\mathscr{L}^{-1} \Delta w^{\alpha}=G_{\alpha}(W) * \Delta W, \\
\Delta V^{\alpha}=\mathscr{L}^{-1} \Delta v^{\alpha}=G_{\alpha}(V) * \Delta V,
\end{gathered}
$$

where $G_{\alpha}=\mathscr{L}^{-1} g_{\alpha}$. For any $\tau \in[0, p]$ according to Lemma 3 we have

$$
\left|G_{\alpha}(V)(\tau)\right| \leqq G_{\alpha}(W)(\tau)
$$

Using Lemma 3 once more we get the inequality

$$
\left|\tilde{F}_{\alpha}(\sigma) * \Delta V^{* \alpha}(\sigma)\right| \leqq \hat{F}_{\alpha}(\sigma) * \Delta W^{* \alpha}(\sigma),
$$


which completes the proof of Lemma 4.

Let us return to Lemma 2. We have the inequalities $\left|Y_{j}(p)\right| \leqq Z_{j}(|p|)$ for all $j \leqq k$ and all $p \in U_{\delta}$ as the assumption of induction. Then (4.11) follows from Lemma 4, where $V_{2}(\sigma)=Y_{k}(p), V_{1}(\sigma)=Y_{k-1}(p), W_{2}(\sigma)=Z_{k}(|p|), W_{1}(\sigma)=Z_{k-1}(|p|)$ and $p=$ $\sigma e^{i \varphi}$.

The vector-valued function $Y_{k+1}(p)$ is holomorphic in $U_{\delta}$ since the series

$$
Y_{k+1}(p)=\sum_{|\alpha| \geqq 0} \tilde{F}_{\alpha}(p) * Y_{k}^{* \alpha}(p)
$$

is majorized by the series

$$
\sum_{|\alpha| \geqq 0} \hat{F}_{\alpha}(|p|) * Z_{k}^{* \alpha}(|p|)
$$

4. The Degenerate Case. In Subsects. 1-3 we proved Theorem 3 in the nondegenerate case. Now let us consider the case when $\lambda_{j}(x)=\lambda_{j} \ln x, j \leqq \ell$ and $\lambda_{j}(x)=$ $\lambda_{j} x, j>\ell$. Here $\ell \in N, \ell<n$ and functions $\lambda_{j}(x)$ are defined by (2.3).

Let us define the Laplace transform of the desired majorizing vector-valued function $Z(p)$ by the system

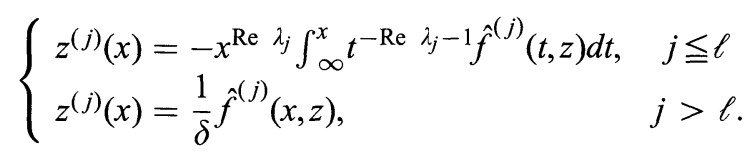

If we rewrite (4.14) as $z(x)=\varphi(x, z(x))$ then

$$
\varphi(\infty, 0)=0, \quad \frac{\partial \varphi}{\partial z}(\infty, 0)=0
$$

follow from (2.2) and from (4.6). Now by means of the arguments of Subsect. 2 one can show that in the degenerate case $Z(p)=\left(\mathscr{L}^{-1} z\right)(p)$ also possesses the properties mentioned in Lemma 1.

If in the first $\ell$ differential equations of (2.3) we make the inverse Laplace transform, differentiate in $p$ and represent the obtained differential equations in the integral form, then we get

$$
Y^{(j)}(p)=p^{-\lambda_{j}-1} \int_{0}^{p} \tau^{\lambda_{J}} \tilde{F}^{(j)}(\tau, Y(\tau)) d \tau, \quad j=1, \ldots, \ell .
$$

The corresponding equations on $Z^{(j)}(p), j=1, \ldots, \ell$, are

$$
Z^{(j)}(p)=p^{-\operatorname{Re} \lambda_{j}-1} \int_{0}^{p} \tau^{\operatorname{Re} \lambda_{J}} \hat{F}^{(j)}(\tau, Z(\tau)) d \tau .
$$

Let $\tilde{H}^{j}(p, Y(p))$ and $\hat{H}^{j}(p, Z(p))$ denote the right-hand sides of the latter equations respectively, $j=1, \ldots, \ell$. Then one can directly check that the inequality

$$
\left|\tilde{F}^{(j)}(\tau, Y(\tau))\right| \leqq \hat{F}^{(j)}(|\tau|, Z(|\tau|))
$$

for all $\tau \in[0, p], p \in U_{\delta}$ implies 


$$
\left|\tilde{H}^{(j)}(p, Y(p))\right| \leqq \hat{H}^{(j)}(|p|, Z(|p|)) .
$$

That means that Lemma 4 can be expanded on the degenerate case. So the remaining part of the proof coincides with that of the nondegenerate case.

5. The Case $v>1$. Let us consider the nondegenerate case. Then the inverse $v$ Laplace transform transfers Eq. (2.1) into

$$
Y(p)=-\frac{1}{v}\left(p^{v}+\frac{\Lambda}{v}\right)^{-1} \tilde{F}(p, Y(p)),
$$

where $\tilde{F}(p, Y(p))=\left[\mathscr{L}_{v}^{-1} \tilde{f}(x, y(x))\right](p)$. A proof of Theorem 3 for $v>1$ in both the nondegenerate and degenerate cases just repeats the corresponding proof for $v=1$ replacing the usual Laplace transform by $v$-Laplace transform.

\section{References}

[Ec] Eckhaus, W.: Singular perturbations of homoclinic orbits in $R^{4}$. SIAM J. Math. Anal. 23, N5, 1269-1290 (1992)

[GJ] Grimshaw, R., Joshi, N.: Weakly non-local solitary waves in a singularly perturbed Korteweg-de Vries equation. SIAM J. Appl. Math., submitted

[Gr] Grimshaw, R.: The use of Borel-summation in the establishment of non-existence of certain travelling-wave solutions of the Kuramoto-Sivashinsky equations. Wave Motion 15, 393395 (1992)

[HM] Hakim, V., Mallick, K.: Exponentially small splitting of separatrices, matching in the complex plane and Borel summation. Nonlinearity 6, N1, 57-70 (1993)

[KO] Kichenassamy, S., Olver, P.J.: Existence and nonexistence of solitary wave solutions to higher-order model evolution equations, SIAM J. Math. Anal. 23, N5, 1141-1166 (1992)

[KS] Kruskal, M., Segur, H.: Asymptotics beyond all orders in a model of crystal growth. Studies in Appl. Math. 85, N2, 129-182 (1991)

[RS] Ramis, J.-P., Sibuya, Y.: Hukukara domains and fundamental existence and uniqueness theorems for asymptotic solutions of Gevrey type. Asymptotic Analysis 2, 39-94 (1989)

[T1] Tovbis, A.: Nonlinear ordinary differential equations resolvable with respect to an irregular singular point. J. Diff. Equat. 107 (1994), to appear

[T2] Tovbis, A.: On exponentially small terms of solutions to nonlinear ordinary differential equations. Methods and Applications of Analysis 1, N1, 57-74 (1994)

[Wa] Wasow, W.: Asymptotic expansions for ordinary differential equations. N.Y.: Dover, 1976 
